\title{
Volume Analysis of Periodontal Bone Specimens Retained in Trephine Burs Using Synchrotron Radiation X-Ray Micro-Computed Tomography
}

\author{
Dongyun Lee $^{1}$, Seob-Gu Kim², Moo-Hyun Cho' ${ }^{1}$, Yong-Gun Kim ${ }^{3, *}$, Jae-Hong Lim ${ }^{2, *}$ \\ 1. Division of Advanced Nuclear Engineering, Pohang University of Science and Technology, Pohang, \\ Korea \\ 2. Pohang Accelerator Laboratory, Pohang University of Science and Technology, Pohang, Korea \\ 3. Department of Periodontology, School of Dentistry, Kyungpook National University, Daegu, Korea \\ * Corresponding authors, periokyg@knu.ac.kr and limjh@postech.ac.kr
}

Bone regeneration studies require accurate volume measurement to evaluate new bone formation. Periodontal specimens, harvested from bone grafting areas inside the oral cavity using trephine burs (TBs), are conventionally analyzed by bone histomorphometry, which estimates volume fractions from photomicrographs of histological sections. Recent development in non-destructive three-dimensional (3D) imaging analysis using X-ray micro-computed tomography $(\mu \mathrm{CT})$ shows potential for replacing the conventional method for accurate volume measurement. We further demonstrated that the use of synchrotron radiation $\mathrm{X}$-ray $\mu \mathrm{CT}(\mathrm{SR}-\mu \mathrm{CT})$ can greatly accelerate high quality data acquisition, easing statistical analysis of multiple specimens [1]. One drawback in $\mu \mathrm{CT}$ sample preparation, which can be avoided in bone histomorphometry, is that specimens need to be ejected from TBs for X-ray transmission. During ejection, specimens may get damaged or even break, which is especially detrimental to weak specimens exhibiting poor bone regeneration. Recently, SR- $\mathrm{CCT}$ using high photon energy X-rays for higher X-ray transmission is becoming more accessible. In this study, we examined the use of SR- $\mu$ CT for volume analysis of intact periodontal bone specimens while they are retained in TBs.

Specimens were prepared as described by Seo, et al. [1]. Bone biopsy was performed 6 months after bone regenerative surgery at dental implant sites using TBs made of TrimRite ${ }^{\circledR}$ stainless steel (TPHB-B3, 3.0$\mathrm{mm}$ outer diameter and 2.3-mm inner diameter; Osung, Kimpo, Korea). Harvested bone specimens retained in TBs were individually transferred to small plastic tubes filled with $4 \%$ neutral-buffered paraformaldehyde. One of the tubes was subjected to SR- $\mu$ CT at beamline 6C Bio Medical Imaging of the Pohang Light Source-II in Pohang, Korea. The entire procedure was reviewed by the Ethics committee of the Kyungpook National University Hospital.

We chose $37 \mathrm{keV}$ for monochromatic X-ray beam illumination from a multilayer monochromator. At that photon energy, X-ray transmission through the tube sample varied from 67\% (through the TB's lateral opening) to $6 \%$ (through the covered side). For CT, the sample was scanned in steps of $0.6^{\circ}$ for a $180^{\circ}$ rotation. Exposure time per projection was $2 \mathrm{~s}$. CT was performed at multiple sample-to-detector distances (SDDs) to examine contrast enhancement caused by phase effect. Detector pixel size was $1.63 \mu \mathrm{m}$. Detector field of view (FOV) was $4.2 \mathrm{~mm}$ horizontally and $3.5 \mathrm{~mm}$ vertically. However, the X-ray beam illumination became narrower in the vertical direction as the photon energy increased, and at $37 \mathrm{keV}$, it was effectively $1.4 \mathrm{~mm}$ full-width-at-half-maximum vertically. CT reconstruction was performed using Octopus 8.7 software implementing the filtered back projection algorithm (XRE, Gent, Belgium). Visualization and volume segmentation was performed using Amira 6.4 (ThermoFisher, Hillsboro, Oregon, USA).

SR- $\mu \mathrm{CT}$ enabled the visualization of the internal structure of a periodontal bone specimen retained in a 
stainless steel TB through its lateral openings. Through a completely covered side of TB, the bone specimen image was poorly reconstructed probably due to insufficient X-ray transmission (data not shown). As shown in Figure 1, reconstructed slice images gradually improved as SDD was increased, indicating that phase contrast played a critical role in image formation. At $120 \mathrm{~mm}$ (Fig. 1a), residual bone was discernable despite the images being very noisy. At $240 \mathrm{~mm}$ (Fig. 1b), residual bone boundaries became distinctive, significantly improving the visibility of residual bone. Bone lacunae were also visible. At $360 \mathrm{~mm}$, the boundaries were further enhanced, and the existence of a discrete, softer phase beside the residual bone was noticed (Fig. 1c, marked by arrows). This phase was believed to be formed by the influx of bone debris into non-mineralized tissue regions during the drilling process, based on the observation that it was denser on the periphery close to the TB's side wall. In all images, noise level was not uniform but locally higher around the specimen-TB boundaries where voxels were the most buried by the TB during CT. This is where X-ray scattering from the TB's metallic body could have exceeded X-ray transmission. X-ray transmission through the TB's covered side was $9 \%$.

Figure 2 presents accompanying image processing and volume analysis of the reconstructed slices at 360 $\mathrm{mm}$ SDD. The non-local means filtering algorithm was applied to clean the images. However, along the specimen-TB boundaries, the resulting grey level was not flat because of inclusion of the scattering noise (Fig. 2a). For easier segmentation, the core volume that was $1.6 \mathrm{~mm}$ in diameter and $1.6 \mathrm{~mm}$ in height was isolated, and the watershed algorithm was applied. The segmented volume of residual bone is visualized in Fig. 2b.

In this study, the potential of SR- $\mu \mathrm{CT}$ for volume analysis of periodontal bone specimens as they are retained in TBs is demonstrated. This should be beneficial because intact volume is examined accurately and non-destructively such that specimens are also spared for further histological analysis. Our results suggest that X-ray transmission through a TB needs to be higher than 9\%; otherwise, X-ray scattering noise from a TB exceeds X-ray transmission signals near a specimen's outer surface, encroaching on available volume for analysis. Strong phase contrast effect is essential for image formation of bone specimens inside TBs, suggesting that this is not easily implemented with $\mu \mathrm{CT}$ using conventional X-ray tube sources. However, the productivity of SR- $\mu \mathrm{CT}$ is considerably hampered because the vertical FOV is limited by the small X-ray beam illumination at $37 \mathrm{keV}$, and exposure time is 10-fold greater than that for uncovered specimens [1]. Development of larger and brighter SR X-ray beam illumination at even higher photon energy is greatly anticipated.

\section{Reference:}

[1] Seo, Bark, Lim and Kim, International Journal of Nanomedicine 10 (2015) p. 129.

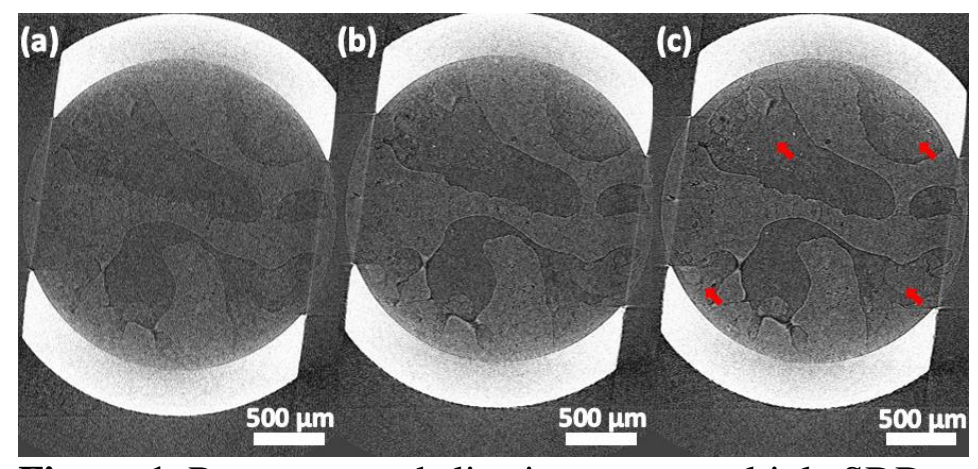

Figure 1. Reconstructed slice images at multiple SDDs (a) $120 \mathrm{~mm}$ SDD; (b) $240 \mathrm{~mm}$ SDD; (c) $360 \mathrm{~mm}$ SDD

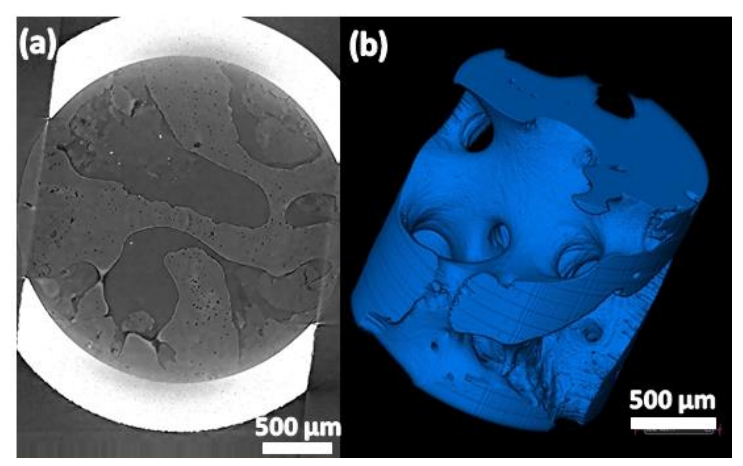

Figure 2. Analysis (a) Noise filtering; (b) 3D-rendered image of residual bone 